\title{
Genetic variability of panicle architecture in traditional finger millets from Koraput valley of Eastern Ghats for crop improvement
}

Debabrata Panda ( $\square$ dpanda80@gmail.com)

Central University of Orissa https://orcid.org/0000-0002-8019-3062

\section{Amrita Tanty}

Central University of Odisha School of Biodiversity and Conservation of Natural Resources

Prafulla K Behera

Central University of Odisha School of Biodiversity and Conservation of Natural Resources

\section{Aloukika Panda}

Central University of Odisha School of Biodiversity and Conservation of Natural Resources

Kartik Lenka

MS Swaminathan Research Foundation

\section{Research Article}

Keywords: Grain yield, Indigenous finger millets, Panicle architecture, Principal component analysis

Posted Date: March 8th, 2021

DOI: https://doi.org/10.21203/rs.3.rs-304691/v1

License: @) (i) This work is licensed under a Creative Commons Attribution 4.0 International License. Read Full License 


\section{Abstract}

Panicle phenotyping is most demanding targets in crop breeding programs as panicle is an important plant parts that influences the grain yield. Diversity of panicle traits were analyzed in 20 traditional finger millet genotypes and three hybrid varieties from Koraput valley of Eastern Ghats. Significant difference of panicle characteristics like panicle length, panicle number, panicle weight, flag leaf area, panicle angle and grain yield were observed in experimented finger millet genotypes. In regards to principal component analysis, the first two axis of principal component obtained $52.97 \%$ of total variation and reflected huge difference between explored genotypes. Highest positive loading was observed for leaf weight followed by panicle number, panicle weight and panicle length and leaf area and are the major determinant for phenotypic variability. All the studied traits showed maximized phenotypic coefficient of variation (PCV) over genotypic variation (GCV) and lower differences found among them. The genotypes showed maximum broad-sense of heritability value for grain yield (82.27\%) and panicle weight (75.96\%) compared to other traits. In addition, genetic advance as mean\% (GAM) ranged from 11.01-29.26\% and high GAM was recorded for panicle angle, leaf area, panicle weight, panicle number and yield. It revealed that these characters can be used as selection criteria in crop improvement program for improving grain yield. Traditional finger millet genotypes such as Bhadi, Murda, Telgu and Chilli recorded superior panicle traits and Limca and Kalia showed better grain yield at par with the hybrids, which can be utilized in future crop improvement program.

\section{Introduction}

Finger millet (Eleusine coracana (L.) Gaertn) is most valuable cereals generally grown on arid and semi-arid regions of the globe under rainfed agro-climatic conditions (Chandra et al. 2016). India is the major producer of finger millet which is cultivated on 1.27 million hectares land brings about 1.89 million tons of yearly production (Sood et al. 2019). Special aspects of millets like the capacity to adjust under extremely unfavorable climatic condition, minimal agroinput requirement and remarkable nutritional value label these crops for future food and nutrition security (Gupta et al. 2017). The crop farming related to various eco-geographical climatic conditions worldwide determines higher genetic variations and perhaps fundamental basis for the future breeding programs (Ceasar et al. 2018). However, the genomic assets accessible for finger millet are limited as contrasted to vital cereals such as rice, wheat and maize which hamper the improvement of this valuable crop (Ceasar et al. 2018). Improvement of finger millet productivity remains the first concern research in majority of the breeding programs intending for climate resilient agriculture. Crop yield is the most important multifaceted traits and is the final outcome of combination of different traits like panicle number, panicle length, grain number and weight of grains etc. (Kumari and Singh 2016; Patil et al. 2018). Panicle characteristics in finger millet are regarded as most intuitive part that controls the quality and yield of finger millet (Wu et al. 2016; Lei et al. 2018). Therefore, panicle architecture is an important component and for the most part makes biggest contribution to grain yield (Zhou et al. 2014; Zhao et al. 2016; Patil et al. 2018). Many genes and QTL responsible for panicle traits and yield associated traits has been reported in finger millet (Saha et al. 2016; Ceasar et al. 2018; Sood et al. 2019). However, in compared to other cereals, relatively less information is reported in finger millets in relation to panicle phenotyping and the relationship between panicle characteristics and grain yield is not yet been explored.

Identifying superior germplasm for increasing productivity of finger millet will assist to acquire food and nutritional security (Ceaser et al. 2018). Earlier studies have been attempted to recognize markers linked to important agronomic and nutritional traits (Mutch et al. 2005; Babu et al. 2014; Kumar et al. 2015; Gimode et al. 2016). However, breeding of finger millet with improved qualities is presently restrained by the absence of efficiently characterized germplasms at the agro-morphological levels (Rajendran et al. 2016; Sood et al. 2019). Previously some attempts has been explored to utilize different morphological and agronomic traits for analyzing genetic diversity of finger millet accessions of 
Africa and Asia (Dida et al. 2008), global finger millet accessions (Babu et al. 2014; Ramakrishnan et al. 2016; Wakista et al. 2017) and genotypes from India (Babu et al. 2018). The genotypes having superior agronomic traits can be utilized in breeding program. Though India holds good numbers of germplasms of finger millet and the majority of the germplasms are collected from the Andhra Pradesh, Karnataka and Tamil Nadu etc. However, very little information is available on genetic variation of traditional finger millet genotypes of Eastern Ghats, particularly from Koraput.

Koraput valley of Eastern Ghats of India is one of the agro-biodiversity hot spots in India and home to a huge number of traditional finger millets (Pradhan et al. 2019). This region with varied agro-climatic conditions surrounded with thick forest and steep mountainous ranges favorable for millet cultivation by local tribes (Panda et al. 2020b). Nowadays due to modern farming practices, these valuable genetic assets are being continuously eroded. The traditional varieties grown by the farmers are likely to be lost shortly if measures are not taken to protect the valuable genetic resources. Recently, some research highlighted the cultivation practice, nutritional and genetic potentiality of finger millets from Koraput (Pradhan et al. 2019; Panda et al. 2020a, b). However, there is limited phenotypic knowledge in regards to panicle phenotyping and yield related traits. Therefore, the objectives of the current study are to elucidate the genetic variation of panicle traits in underutilized finger millet genotypes from Koraput valley and to elucidate the genetic parameters such as heritability and genetic advance which will be useful for future breeding program.

\section{Materials And Methods}

\section{Experimental material and growth condition}

Twenty different traditional finger millet genotypes and three hybrid varieties (GPU66, ML365 and Bhairabi) popularly cultivated from Koraput valley of Eastern Ghats were selected for the study. The details of finger millet genotypes with their maturity duration and special characteristics are presented in Table 1. 
Table 1

Details of finger millet genotypes with their maturity duration, morphological variation of seed colour and panicle type.

\begin{tabular}{|c|c|c|c|c|c|}
\hline $\begin{array}{l}\text { SI } \\
\text { No. }\end{array}$ & $\begin{array}{l}\text { Variety } \\
\text { name }\end{array}$ & Origin of Finger millet & $\begin{array}{l}\text { Maturity } \\
\text { (days) }\end{array}$ & $\begin{array}{l}\text { Seed } \\
\text { colour }\end{array}$ & Panicle type \\
\hline 1 & Bada & Local landrace of Koraput & $90-110$ & $\begin{array}{l}\text { Reddish } \\
\text { brown }\end{array}$ & Semi-compact finger \\
\hline 2 & Bhadi & Local landrace of Koraput & $\begin{array}{l}110- \\
115\end{array}$ & $\begin{array}{l}\text { Dark } \\
\text { brown }\end{array}$ & Open finger \\
\hline 3 & Biri & Local landrace of Koraput & $90-100$ & $\begin{array}{l}\text { Reddish } \\
\text { brown }\end{array}$ & Droopy finger \\
\hline 4 & Chilli & Local landrace of Koraput & $\begin{array}{l}110- \\
120\end{array}$ & $\begin{array}{l}\text { Reddish } \\
\text { brown }\end{array}$ & Droopy finger \\
\hline 5 & Chillikangra & Local landrace of Koraput & $90-110$ & $\begin{array}{l}\text { Orange } \\
\text { red }\end{array}$ & Semi-compact finger \\
\hline 6 & Dasara & Local landrace of Koraput & $80-90$ & Brown & Fist type finger \\
\hline 7 & Jama & Local landrace of Koraput & $\begin{array}{l}100- \\
105\end{array}$ & $\begin{array}{l}\text { Light } \\
\text { brown }\end{array}$ & Compact finger \\
\hline 8 & Jhana & Local landrace of Koraput & $\begin{array}{l}110- \\
125\end{array}$ & Brown & Semi-compact finger \\
\hline 9 & Kalia & Local landrace of Koraput & $\begin{array}{l}105- \\
115\end{array}$ & $\begin{array}{l}\text { Light } \\
\text { brown }\end{array}$ & Semi-compact finger \\
\hline 10 & Kurkuti & Local landrace of Koraput & $\begin{array}{l}115- \\
125\end{array}$ & $\begin{array}{l}\text { Reddish } \\
\text { brown }\end{array}$ & Semi-compact finger \\
\hline 11 & Ladu & Local landrace of Koraput & $\begin{array}{l}110- \\
120\end{array}$ & $\begin{array}{l}\text { Reddish } \\
\text { brown }\end{array}$ & Semi-compact finger \\
\hline 12 & Lala & Local landrace of Koraput & $\begin{array}{l}100- \\
110\end{array}$ & Brown & Open finger \\
\hline 13 & Limca & Local landrace of Koraput & $\begin{array}{l}110- \\
120\end{array}$ & $\begin{array}{l}\text { Reddish } \\
\text { broen }\end{array}$ & Semi-compact \\
\hline 14 & Kalua & Local landrace of Koraput & $\begin{array}{l}105- \\
110\end{array}$ & Brown & Compact finger \\
\hline 15 & Mami & Local landrace of Koraput & $\begin{array}{l}110- \\
120\end{array}$ & Brown & $\begin{array}{l}\text { Semi-compact type } \\
\text { finger }\end{array}$ \\
\hline 16 & Murda & Local landrace of Koraput & $\begin{array}{l}100- \\
110\end{array}$ & $\begin{array}{l}\text { Reddish } \\
\text { brown }\end{array}$ & Open type finger \\
\hline 17 & Muskuri & Local landrace of Koraput & $\begin{array}{l}110- \\
120\end{array}$ & $\begin{array}{l}\text { Dark } \\
\text { brown }\end{array}$ & Open finger \\
\hline 18 & Sillijanha & Local landrace of Koraput & $\begin{array}{l}100- \\
110\end{array}$ & Brown & Semi-compact finger \\
\hline 19 & Taya & Local landrace of Koraput & $\begin{array}{l}115- \\
120\end{array}$ & $\begin{array}{l}\text { Dark } \\
\text { brown }\end{array}$ & Semi-compact finger \\
\hline 20 & Telugu & Local landrace of Koraput & $\begin{array}{l}100- \\
110\end{array}$ & $\begin{array}{l}\text { Orange } \\
\text { red }\end{array}$ & Open type finger \\
\hline
\end{tabular}




\begin{tabular}{|clllll|}
\hline $\begin{array}{l}\text { SI } \\
\text { No. }\end{array}$ & $\begin{array}{l}\text { Variety } \\
\text { name }\end{array}$ & Origin of Finger millet & $\begin{array}{l}\text { Maturity } \\
\text { (days) }\end{array}$ & $\begin{array}{l}\text { Seed } \\
\text { colour }\end{array}$ & Panicle type \\
\hline 21 & GPU 66 & $\begin{array}{l}\text { Hybrid variety (PR 202 x GPU 28) from } \\
\text { PC Unit, Bangalore, Karnataka }\end{array}$ & $\begin{array}{l}112- \\
115\end{array}$ & Brown & $\begin{array}{l}\text { Medium compact ears } \\
\text { with tip incurved fingers }\end{array}$ \\
\hline 22 & ML 365 & $\begin{array}{l}\text { Developed and released by University of } \\
\text { Agricultural Science, Bangalore }\end{array}$ & $\begin{array}{l}110- \\
115\end{array}$ & Brown & $\begin{array}{l}\text { Semi compact ears with } \\
\text { tip incurved fingers }\end{array}$ \\
\hline 23 & Bhairabi & $\begin{array}{l}\text { Mutant of Budha Mandia from OUAT, } \\
\text { Bhubaneswar, Odisha }\end{array}$ & $\begin{array}{l}105- \\
110\end{array}$ & $\begin{array}{l}\text { Orange } \\
\text { red }\end{array}$ & Compact type finger \\
\hline
\end{tabular}

The finger millet genotypes are grown in the experiment garden of Regional Center of Swaminathan Research Foundation, Koraput during Kharif season in three replications with randomized block design. Healthy seeds of all the genotypes were selected shown in the nursery bed. Experiment field was prepared by adding organic fertilizer in the form of farmyard manure. After 25 days of growth 3 seedlings per hill was transplanted in the area of one $\mathrm{m}^{-2}$ with 20 $\mathrm{cm} \times 15 \mathrm{~cm}$ spacing between the hill. The plants are regularly irrigated with running water. The maximum light intensity, air temperature and humidity were recorded daily basis and were $1310 \pm 60 \mu \mathrm{mol} \mathrm{m} \mathrm{m}^{-2} \mathrm{~s}^{-1}, 33.5 \pm 3^{\circ} \mathrm{C}$ and $60-70 \%$, respectively.

\section{Phenotypic Characterization}

Three different panicles were collected from healthy seedlings during the maturity stage (20th to 30th December 2019). The panicles were cut down through a sharp scissor about $1 \mathrm{~cm}$ below from first leaf during collection. The panicle samples were photographed by using a DSLR camera and brought to the laboratory for measurement of panicle traits.

Panicle numbers of each plant were counting by total branch number emerged per hill. Angle between leaf and panicles of individual sample was determined by using a protractor during collection time. Leaf area was determined by measuring length and width of leaf through a measuring scale and further calculated following Yoshida et al. (1976) formula [Leaf Area = length $\times$ width $\times 0.67 \mathrm{~cm}^{2}$ ]. Scientific ruler was used to determine panicle length from $1 \mathrm{~cm}$ down of flag leaf to the tip. Dry weight of leaf and panicle were obtained by oven dried $\left(72{ }^{\circ} \mathrm{C}\right)$ of individual sample around $48 \mathrm{~h}$ and subsequent weighing using digital electronic balance. After harvesting of total plot, the grain weight was noted and grain yield of each genotype was expressed in $\mathrm{t} \mathrm{per} \mathrm{ha}^{-1}$.

\section{Measurement Of Genetic Parameters}

Genetic variability parameters such as variance at genetic level $\left(\sigma_{G}^{2}\right)$ and variance at phenotyping level $\left(\sigma^{2}\right)$ were calculated following the formula of Steel et al. (1997). Then phenotypic coefficient of variance (PCV), genotypic coefficient of variation (GCV) and environmental coefficient of variation (ECV) were determined according to Burton and Devane (1953). By taking the PCV and GCV value, heritability $\left(\mathrm{h}^{2}{ }_{\mathrm{bs}}\right)$ was calculated following Falconer and Mackay (1996). Further, as per Johnson et al. (1955) genetic advance (GA) and genetic advance as mean\% (GAM) were calculated.

\section{Statistical analysis}

Different parameters were recorded triplicate and one-way ANOVA was performed by applying CropStat-7.2 software. Multiple correlation and multivariate analysis (Principal component analysis and Cluster analysis) were carried out by 
PAST-3 software. Based on the panicle phenotyping parameters, dendrogarm exhibiting Bray-Curtis similarity index among studied finger millets were constructed using UPGMA cluster analysis.

\section{Results}

\section{Diversity of panicle traits}

Morphological diversity of panicles in studied finger millets were depicted in Fig. 1. Variation of different morphological characteristics like panicle number, panicle length, panicle weight, leaf area, leaf weight, panicle weight and grain yield were shown in Table 2. The summary statistics of various panicles attributes in finger millet genotypes were shown in Table 3. The coefficient of variation (CV) of different panicle traits were ranged from $4.501-25.450 \%$ among the finger millet genotypes. The maximal CV \% was noticed in panicle angle $(25.45 \%)$ followed by leaf area (23.60\%), panicle number (19.81\%), panicle weight (17.21\%), grain yield (13.59\%), panicle length (10.10\%) and lowest CV was observed in leaf weight (4.501\%). The ANOVA analysis also clearly showed that remarkable variation between genotypes for studied panicle traits (Table 3 ). The value of variance was highest in panicle angle (31.2\%) followed by leaf area (8.51\%), panicle length (9.0\%) and lowest variance was observed in leaf weight (0.003\%). 
Table 2

Variation of panicle traits in studied finger millet genotypes of Koraput. Data are mean of three replications \pm SD.

Same alphabet in a column are not significantly different at $P<0.05$.

\begin{tabular}{|c|c|c|c|c|c|c|c|}
\hline Variety & $\begin{array}{l}\text { Panicle } \\
\text { No }\end{array}$ & $\begin{array}{l}\text { Panicle } \\
\text { length } \\
\text { (cm) }\end{array}$ & $\begin{array}{l}\text { Panicle angle } \\
\text { (degree) }\end{array}$ & $\begin{array}{l}\text { Leaf area } \\
\left(\mathrm{cm}^{-2}\right)\end{array}$ & $\begin{array}{l}\text { Leaf } \\
\text { weight } \\
\left(\text { g leaf }^{-1}\right)\end{array}$ & $\begin{array}{l}\text { Panicle } \\
\text { weight } \\
\text { (g Panicle } \\
\text { 1) }\end{array}$ & $\begin{array}{l}\text { Yield } \\
\left(\mathrm{t} \mathrm{ha}^{-1}\right)\end{array}$ \\
\hline Bada & $\begin{array}{l}6.50 \pm \\
0.71^{a}\end{array}$ & $\begin{array}{l}33.50 \pm \\
0.99^{a}\end{array}$ & $25.00 \pm 7.07^{a}$ & $\begin{array}{l}11.27 \pm \\
2.96^{b}\end{array}$ & $\begin{array}{l}1.17 \pm \\
0.07^{b}\end{array}$ & $\begin{array}{l}11.20 \pm \\
1.24^{a}\end{array}$ & $\begin{array}{l}2.14 \pm \\
0.03^{d}\end{array}$ \\
\hline Bhadi & $\begin{array}{l}7.00 \pm \\
1.41^{a}\end{array}$ & $\begin{array}{l}31.75 \pm \\
1.06^{a}\end{array}$ & $25.00 \pm 7.07^{a}$ & $\begin{array}{l}6.95 \pm \\
2.67^{c}\end{array}$ & $\begin{array}{l}1.08 \pm \\
0.03^{c}\end{array}$ & $\begin{array}{l}8.78 \pm 1.09 \\
c\end{array}$ & $\begin{array}{l}1.89 \pm \\
0.01^{\mathrm{e}}\end{array}$ \\
\hline Biri & $\begin{array}{l}4.00 \pm \\
0.00^{b}\end{array}$ & $\begin{array}{l}34.45 \pm \\
3.46^{a}\end{array}$ & $27.50 \pm 3.54^{a}$ & $\begin{array}{l}13.47 \pm \\
1.31^{b}\end{array}$ & $\begin{array}{l}1.25 \pm \\
0.12^{a}\end{array}$ & $\begin{array}{l}11.57 \pm \\
0.36^{a}\end{array}$ & $\begin{array}{l}1.45 \pm \\
0.07^{\mathrm{e}}\end{array}$ \\
\hline Chilli & $\begin{array}{l}4.50 \pm \\
0.71^{b}\end{array}$ & $\begin{array}{l}31.60 \pm \\
3.11^{a}\end{array}$ & $20.00 \pm 0.00^{b}$ & $\begin{array}{l}14.55 \pm \\
0.79^{a}\end{array}$ & $\begin{array}{l}1.22 \pm \\
0.02^{a}\end{array}$ & $\begin{array}{l}13.06 \pm \\
0.03^{a}\end{array}$ & $\begin{array}{l}1.92 \pm \\
0.06\end{array}$ \\
\hline Chillikangra & $\begin{array}{l}5.00 \pm \\
1.41^{b}\end{array}$ & $\begin{array}{l}34.35 \pm \\
1.06^{a}\end{array}$ & $15.00 \pm 0.00^{b}$ & $\begin{array}{l}12.62 \pm \\
1.08^{b}\end{array}$ & $\begin{array}{l}1.19 \pm \\
0.00^{b}\end{array}$ & $\begin{array}{l}7.64 \pm 0.61 \\
c\end{array}$ & $\begin{array}{l}1.92 \pm \\
0.03^{\mathrm{e}}\end{array}$ \\
\hline Dasara & $\begin{array}{l}3.50 \pm \\
0.71^{b}\end{array}$ & $\begin{array}{l}25.25 \pm \\
4.74^{b}\end{array}$ & $30.00 \pm 7.07^{a}$ & $\begin{array}{l}9.45 \pm \\
2.27^{c}\end{array}$ & $\begin{array}{l}1.14 \pm \\
0.05^{c}\end{array}$ & $\begin{array}{l}9.26 \pm 1.09 \\
b\end{array}$ & $\begin{array}{l}1.85 \pm \\
0.07^{\mathrm{e}}\end{array}$ \\
\hline Jama & $\begin{array}{l}4.00 \pm \\
0.00^{b}\end{array}$ & $\begin{array}{l}29.35 \pm \\
0.64^{b}\end{array}$ & $10.00 \pm 0.00^{c}$ & $\begin{array}{l}11.26 \pm \\
2.11^{b}\end{array}$ & $\begin{array}{l}1.23^{ \pm} \\
0.06^{a}\end{array}$ & $\begin{array}{l}9.36 \pm 1.42 \\
b\end{array}$ & $\begin{array}{l}2.05 \pm \\
0.07^{d}\end{array}$ \\
\hline Jhana & $\begin{array}{l}5.50 \pm \\
0.71^{b}\end{array}$ & $\begin{array}{l}30.90 \pm \\
1.27^{a}\end{array}$ & $15.00 \pm 7.07^{b}$ & $\begin{array}{l}14.18 \pm \\
6.86^{a}\end{array}$ & $\begin{array}{l}1.23 \pm \\
0.04^{a}\end{array}$ & $\begin{array}{l}12.08 \pm \\
0.14^{a}\end{array}$ & $\begin{array}{l}1.82 \pm \\
0.05^{\mathrm{e}}\end{array}$ \\
\hline Kalia & $\begin{array}{l}5.00 \pm \\
1.41^{b}\end{array}$ & $\begin{array}{l}28.15 \pm \\
0.92^{b}\end{array}$ & $17.50 \pm 3.54^{b}$ & $\begin{array}{l}9.33^{ \pm} \\
0.57^{c}\end{array}$ & $\begin{array}{l}1.18 \pm \\
0.12^{b}\end{array}$ & $\begin{array}{l}11.93 \pm \\
2.41^{a}\end{array}$ & $\begin{array}{l}2.20 \pm \\
0.05^{d}\end{array}$ \\
\hline Kurkuti & $\begin{array}{l}5.50 \pm \\
0.71^{b}\end{array}$ & $\begin{array}{l}33.65 \pm \\
4.74^{a}\end{array}$ & $22.50 \pm 3.54^{\mathrm{a}}$ & $\begin{array}{l}14.38 \pm \\
1.75^{a}\end{array}$ & $\begin{array}{l}1.18 \pm \\
0.04^{b}\end{array}$ & $\begin{array}{l}11.66 \pm \\
2.17^{a}\end{array}$ & $\begin{array}{l}1.92 \pm \\
0.06^{\mathrm{e}}\end{array}$ \\
\hline Ladu & $\begin{array}{l}6.00 \pm \\
0.00^{a}\end{array}$ & $\begin{array}{l}28.05 \pm \\
2.62^{b}\end{array}$ & $27.50 \pm 3.54^{a}$ & $\begin{array}{l}13.75 \pm \\
1.71^{a}\end{array}$ & $\begin{array}{l}1.16 \pm \\
0.00^{b}\end{array}$ & $\begin{array}{l}10.56 \pm \\
0.97^{b}\end{array}$ & $\begin{array}{l}2.13 \pm \\
0.05^{d}\end{array}$ \\
\hline Lala & $\begin{array}{l}4.00 \pm \\
0.00^{b}\end{array}$ & $\begin{array}{l}30.45 \pm \\
4.60^{a}\end{array}$ & $22.50 \pm 3.54^{a}$ & $\begin{array}{l}17.06 \pm \\
4.06^{a}\end{array}$ & $\begin{array}{l}1.18 \pm \\
0.01^{a}\end{array}$ & $\begin{array}{l}10.01 \pm \\
1.19 b\end{array}$ & $\begin{array}{l}1.61 \pm \\
0.044^{\mathrm{e}}\end{array}$ \\
\hline Limca & $\begin{array}{l}5.50 \pm \\
0.71^{b}\end{array}$ & $\begin{array}{l}28.15 \pm \\
2.33^{b}\end{array}$ & $22.50 \pm 3.54^{a}$ & $\begin{array}{l}14.17 \pm \\
0.03^{a}\end{array}$ & $\begin{array}{l}1.19 \pm \\
0.01^{a}\end{array}$ & $\begin{array}{l}10.25 \pm \\
0.55^{b}\end{array}$ & $\begin{array}{l}2.33 \pm \\
0.04^{c}\end{array}$ \\
\hline Kalua & $\begin{array}{l}4.50 \pm \\
0.71^{b}\end{array}$ & $\begin{array}{l}26.60 \pm \\
2.55^{b}\end{array}$ & $30.00 \pm 14.14^{a}$ & $\begin{array}{l}14.24 \pm \\
3.48^{a}\end{array}$ & $\begin{array}{l}1.14 \pm \\
0.02^{b}\end{array}$ & $\begin{array}{l}8.74 \pm 0.76 \\
c\end{array}$ & $\begin{array}{l}1.93 \pm \\
0.11^{\mathrm{e}}\end{array}$ \\
\hline Mami & $\begin{array}{l}5.00 \pm \\
1.41^{b}\end{array}$ & $\begin{array}{l}23.95 \pm \\
1.06^{c}\end{array}$ & $22.50 \pm 3.54^{a}$ & $\begin{array}{l}7.22 \pm \\
0.36^{c}\end{array}$ & $\begin{array}{l}1.12 \pm \\
0.00^{b}\end{array}$ & $\begin{array}{l}9.61 \pm 0.63 \\
b\end{array}$ & $\begin{array}{l}1.81 \pm \\
0.04^{\mathrm{e}}\end{array}$ \\
\hline Murda & $\begin{array}{l}7.00 \pm \\
1.41^{\mathrm{a}}\end{array}$ & $\begin{array}{l}27.50 \pm \\
3.25^{b}\end{array}$ & $22.50 \pm 3.54^{a}$ & $\begin{array}{l}12.31 \pm \\
2.26^{b}\end{array}$ & $\begin{array}{l}1.13 \pm \\
0.01^{b}\end{array}$ & $\begin{array}{l}10.90 \pm \\
2.32^{b}\end{array}$ & $\begin{array}{l}2.04 \pm \\
0.04\end{array}$ \\
\hline Muskuri & $\begin{array}{l}6.00 \pm \\
0.00^{a}\end{array}$ & $\begin{array}{l}25.95 \pm \\
0.49^{b}\end{array}$ & $27.50 \pm 3.54^{a}$ & $\begin{array}{l}12.48 \pm \\
0.45^{b}\end{array}$ & $\begin{array}{l}1.14 \pm \\
0.03^{b}\end{array}$ & $\begin{array}{l}8.30 \pm 0.36 \\
c\end{array}$ & $\begin{array}{l}1.74 \pm \\
0.04\end{array}$ \\
\hline
\end{tabular}




\begin{tabular}{|c|c|c|c|c|c|c|c|}
\hline Variety & $\begin{array}{l}\text { Panicle } \\
\text { No }\end{array}$ & $\begin{array}{l}\text { Panicle } \\
\text { length } \\
\text { (cm) }\end{array}$ & $\begin{array}{l}\text { Panicle angle } \\
\text { (degree) }\end{array}$ & $\begin{array}{l}\text { Leaf area } \\
\left(\mathrm{cm}^{-2}\right)\end{array}$ & 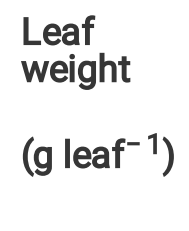 & $\begin{array}{l}\text { Panicle } \\
\text { weight } \\
\text { (g Panicle- } \\
\text { 1) }\end{array}$ & $\begin{array}{l}\text { Yield } \\
\left(\mathrm{tha} \mathrm{a}^{-1}\right)\end{array}$ \\
\hline Sillijanha & $\begin{array}{l}6.00 \pm \\
1.41^{a}\end{array}$ & $\begin{array}{l}29.75 \pm \\
1.48^{b}\end{array}$ & $15.00 \pm 7.07^{b}$ & $\begin{array}{l}12.06 \pm \\
4.31^{b}\end{array}$ & $\begin{array}{l}1.19 \pm \\
0.09^{a}\end{array}$ & $\begin{array}{l}10.43 \pm \\
0.92^{b}\end{array}$ & $\begin{array}{l}1.86 \pm \\
0.02 \mathrm{e}\end{array}$ \\
\hline Taya & $\begin{array}{l}4.50 \pm \\
0.71^{b}\end{array}$ & $\begin{array}{l}28.05 \pm \\
2.76^{b}\end{array}$ & $17.50 \pm 3.54^{b}$ & $\begin{array}{l}12.37 \pm \\
5.05^{b}\end{array}$ & $\begin{array}{l}1.17 \pm \\
0.06^{b}\end{array}$ & $\begin{array}{l}9.00 \pm 0.79 \\
c\end{array}$ & $\begin{array}{l}2.02 \pm \\
0.02 d\end{array}$ \\
\hline Telugu & $\begin{array}{l}4.00 \pm \\
0.00^{b}\end{array}$ & $\begin{array}{l}30.60 \pm \\
3.82^{a}\end{array}$ & $17.50 \pm 3.54^{b}$ & $\begin{array}{l}9.10 \pm \\
0.59^{c}\end{array}$ & $\begin{array}{l}1.20 \pm \\
0.01^{a}\end{array}$ & $\begin{array}{l}13.08 \pm \\
0.41^{a}\end{array}$ & $\begin{array}{l}2.09 \pm \\
0.05^{d}\end{array}$ \\
\hline Gpu 66 & $\begin{array}{l}5.00 \pm \\
1.41^{\mathrm{b}}\end{array}$ & $\begin{array}{l}26.70 \pm \\
5.23^{b}\end{array}$ & $30.00 \pm 0.00^{a}$ & $\begin{array}{l}10.62 \pm \\
0.70^{c}\end{array}$ & $\begin{array}{l}1.12^{ \pm} \\
0.02^{c}\end{array}$ & $\begin{array}{l}10.30 \pm \\
2.28^{b}\end{array}$ & $\begin{array}{l}2.23 \pm \\
0.04^{c}\end{array}$ \\
\hline Ml365 & $\begin{array}{l}4.00 \pm \\
1.41^{b}\end{array}$ & $\begin{array}{l}32.45 \pm \\
2.47^{a}\end{array}$ & $25.00 \pm 7.07^{a}$ & $\begin{array}{l}19.64 \pm \\
1.40^{a}\end{array}$ & $\begin{array}{l}1.34 \pm \\
0.07^{a}\end{array}$ & $\begin{array}{l}14.88 \pm \\
0.37^{a}\end{array}$ & $\begin{array}{l}2.44 \pm \\
0.02^{b}\end{array}$ \\
\hline Bhairabi & $\begin{array}{l}4.50 \pm \\
0.71^{b}\end{array}$ & $\begin{array}{l}31.90 \pm \\
1.84^{a}\end{array}$ & $17.50 \pm 3.54^{b}$ & $\begin{array}{l}11.78 \pm \\
0.34 \mathrm{~b}\end{array}$ & $\begin{array}{l}1.19 \pm \\
0.01^{b}\end{array}$ & $\begin{array}{l}8.06 \pm 0.17 \\
c\end{array}$ & $\begin{array}{l}2.72 \pm \\
0.03^{a}\end{array}$ \\
\hline Mean & 5.06 & 29.69 & 21.95 & 12.35 & 1.18 & 10.46 & 2.00 \\
\hline $\begin{array}{l}\operatorname{LSD}(P< \\
0.05)\end{array}$ & 1.91 & 5.02 & 10.8 & 5.6 & 0.106 & 2.53 & 0.104 \\
\hline
\end{tabular}

Table 3

Summary statistics of different panicle traits in studied finger millet genotypes of Koraput. ** significance at 0.01 level of probability.

\begin{tabular}{|llllllll|}
\hline $\begin{array}{l}\text { Statistical } \\
\text { parameters }\end{array}$ & $\begin{array}{l}\text { Panicle } \\
\text { number }\end{array}$ & $\begin{array}{l}\text { Panicle } \\
\text { length }(\mathbf{c m})\end{array}$ & $\begin{array}{l}\text { Panicle } \\
\text { angle }\end{array}$ & $\begin{array}{l}\text { Leaf } \\
\text { area } \\
\left(\mathbf{c m}^{-2}\right)\end{array}$ & $\begin{array}{l}\text { Leaf } \\
\text { weight }\end{array}$ & $\begin{array}{l}\text { Panicle weight (g } \\
\left.\text { Panicle }^{-1}\right)\end{array}$ & $\begin{array}{l}\text { Yield }(\mathbf{t} \\
\left.\text { ha }^{-1}\right)\end{array}$ \\
\hline Number & 23 & 23 & 23 & 23 & 23 & 23 & 23 \\
\hline Minimum & 3.500 & 23.95 & 10.00 & 6.955 & 1.085 & 7.637 & 1.450 \\
\hline Maximum & 7.000 & 34.45 & 30.00 & 19.644 & 1.338 & 14.879 & 2.720 \\
\hline Sum & 116.500 & 683.05 & 505.00 & 284.268 & 27.138 & 240.670 & 46.055 \\
\hline Mean & 5.065 & 29.698 & 21.957 & 12.359 & 1.180 & 10.464 & 2.002 \\
\hline Standard error & 0.209 & 0.626 & 1.165 & 0.608 & 0.011 & 0.376 & 0.057 \\
\hline Variance & 1.007 & 9.000 & 31.225 & 8.511 & 0.003 & 3.243 & 0.074 \\
\hline $\begin{array}{l}\text { Standard } \\
\text { deviation }\end{array}$ & 1.003 & 3.000 & 5.588 & 2.917 & 0.053 & 1.801 & 0.272 \\
\hline CV (\%) & 19.811 & 10.102 & 25.450 & 23.604 & 4.501 & 17.211 & 13.590 \\
\hline Fvalue & $2.39 * *$ & $2.14 * *$ & $2.27 * *$ & $2.23 * *$ & $2.13 * *$ & $4.14 * *$ \\
\hline
\end{tabular}


Panicle number remarkably $(P<0.05)$ changed from 3.5 in Dasara to 7.0 in Murda and Bhadi having average value of 5.06 across the studied finger millet genotypes (Table 2). Panicle length notably $(P<0.05)$ ranged from $23.95 \mathrm{~cm}$ (Mami) to $34.45 \mathrm{~cm}$ (Biri) having mean estimation of $29.69 \mathrm{~cm}$ across the genotypes. Panicle angle varied from $10^{\circ}$ to $30^{\circ}$ across the genotypes and maximum panicle angle was noted in 'Dasara' and 'Kalua' landraces (Table 2). Leaf

area was significantly $(P<0.05)$ ranged from $6.955 \mathrm{~cm}^{2}$ in 'Bhadi' to $19.64 \mathrm{~cm}^{2}$ in 'ML 365' with a mean value of 12.35 $\mathrm{cm}^{2}$. Similarly, significant $(P<0.05)$ difference of leaf weight were found among genotypes and varied from $1.08 \mathrm{~g}$ leaf $^{-1}$ in 'Bhadi' landrace to $1.34 \mathrm{~g} \mathrm{leaf}^{-1}$ in 'ML 365'. Panicle weight was remarkably $(P<0.05)$ varied among examined genotypes and hybrid variety 'ML 365' showed highest panicle weight of $14.88 \mathrm{~g}$ panicle ${ }^{-1}$ whereas 'Chillikangra' showed lowest panicle weight of $7.64 \mathrm{~g} \mathrm{panicle}^{-1}$ (Table 2). Significant $(P<0.05)$ difference of grain yield were recorded among the studied finger millet genotypes. The hybrid variety 'Bhairabi' showed highest yield 2.72 $\mathrm{t} \mathrm{ha}^{-1}$, whereas 'Limca' genotype showed the lowest yield of $1.45 \mathrm{t} \mathrm{ha}^{-1}$ with a mean value of $2.00 \mathrm{t} \mathrm{ha}^{-1} \mathrm{across}$ the genotypes (Table 2).

\section{Genetic Variability Parameters}

Genetic variability of studied morphological traits was shown in Table 4. All the studied traits showed higher phenotypic variation $\left(\sigma^{2} \mathrm{P}\right)$ than that of genotypic variation $\left(\sigma^{2}{ }_{G}\right)$. In addition, PCV was $>$ GCV and ECV in studied panicle traits and lower differences found among them (Table 4). The trait showed the largest PCV data for panicle angle (25.34\%) next to leaf area (23.15\%) and lowest PCV data was obtained for panicle length (9.957\%). Similarly, GCV value varied from $7.29-18.97 \%$ and panicle angle showed highest GCV (\%) accounts for 18.97\% (Table 4). The broad sense of heritability $\left(\mathrm{h}^{2}\right.$ bs $)$ varied from $50-82.270 \%$ and the highest $\mathrm{h}^{2}$ bs value was observed for grain yield (82.270\%) followed by panicle weight (75.968\%) among the finger millets. The highest genetic advance (GA) was recorded for panicle angle (6.424\%). The GAM value varied from 11.007-29.269\% and maximum GAM was recorded for panicle angle $(29.269 \%)$ followed by leaf area $(26.419 \%)$, panicle weight $(26.342 \%)$, panicle number $(23.733 \%)$ and yield $(22.477 \%)$ (Table 4$)$. 
Table 4

Genetic variability parameters such as range, mean, standard error $(\mathrm{SE})$, genotypic variation $\left(\sigma_{\mathrm{G}}^{2}\right)$, phenotypic variation $\left(\sigma^{2} \mathrm{P}\right)$, environmental coefficient of variation (ECV), genotypic coefficient of variation (GCV), phenotypic coefficient of variation (PCV), heritability in broad sense $\left(\mathrm{h}^{2}\right.$ bs $)$, genetic advance (GA) and genetic advance as percentage of the mean (GAM) for different traits in finger millet genotypes of Koraput.

\begin{tabular}{|c|c|c|c|c|c|c|c|c|c|c|}
\hline Parameters & Range & $\begin{array}{l}\text { Mean } \\
\pm \text { SE }\end{array}$ & $\sigma_{G}^{2}$ & $\sigma_{P}^{2}$ & $\begin{array}{l}\text { ECV } \\
(\%)\end{array}$ & $\begin{array}{l}\text { GCV } \\
(\%)\end{array}$ & $\begin{array}{l}\text { PCV } \\
(\%)\end{array}$ & $\begin{array}{l}h^{2}{ }_{b s} \\
(\%)\end{array}$ & GA & GAM \\
\hline $\begin{array}{l}\text { Panicle } \\
\text { number }\end{array}$ & $\begin{array}{l}3.50- \\
7.00\end{array}$ & $\begin{array}{l}5.065 \\
\pm 0.209\end{array}$ & 0.585 & 1.005 & 18.095 & 15.101 & 19.793 & 58.209 & 1.202 & 23.733 \\
\hline $\begin{array}{l}\text { Panicle } \\
\text { length }\end{array}$ & $\begin{array}{l}23.95- \\
34.45\end{array}$ & $\begin{array}{l}29.690 \\
\pm 0.626\end{array}$ & 4.690 & 8.740 & 9.586 & 7.294 & 9.957 & 53.661 & 3.268 & 11.007 \\
\hline $\begin{array}{l}\text { Panicle } \\
\text { angle }\end{array}$ & $\begin{array}{l}10.0- \\
30.0\end{array}$ & $\begin{array}{l}21.950 \\
\pm 1.165\end{array}$ & 17.350 & 30.950 & 23.760 & 18.976 & 25.345 & 56.058 & 6.424 & 29.269 \\
\hline Leaf area & $\begin{array}{l}6.95- \\
19.60\end{array}$ & $\begin{array}{l}12.350 \\
\pm 0.608\end{array}$ & 4.530 & 8.180 & 21.877 & 17.234 & 23.158 & 55.379 & 3.263 & 26.419 \\
\hline $\begin{array}{l}\text { Leaf } \\
\text { weight }\end{array}$ & $\begin{array}{l}1.08- \\
1.34\end{array}$ & $\begin{array}{l}1.179 \\
\pm 0.011\end{array}$ & 0.013 & 0.026 & 13.676 & 9.671 & 13.676 & 50.000 & 0.166 & 14.087 \\
\hline $\begin{array}{l}\text { Panicle } \\
\text { weight }\end{array}$ & $\begin{array}{l}7.64- \\
14.87\end{array}$ & $\begin{array}{l}10.460 \\
\pm 0.376\end{array}$ & 2.355 & 3.100 & 11.670 & 14.671 & 16.833 & 75.968 & 2.755 & 26.342 \\
\hline Yield & $\begin{array}{l}1.45- \\
2.72\end{array}$ & $\begin{array}{l}2.002 \\
\pm 0.057\end{array}$ & 0.058 & 0.071 & 7.898 & 12.030 & 13.263 & 82.270 & 0.450 & 22.477 \\
\hline
\end{tabular}

\section{Principal Component Analysis}

Variations of panicle traits among finger millet genotypes were evaluated by multivariate analysis including PCA (Table 5; Fig. 2). All the studied parameters were separated into seven principal components (PC), among which, three PC having Eigen value $>1$ (Table 4). In PC 1 the highest positive loading was found for leaf weight (0.588) succeeded by leaf area (0.442), panicle weight $(0.424)$ and panicle length $(0.390)$ with a variance of $38.06 \%$, whereas in PC 2 maximal variability was marked in panicle angle (0.726) and leaf area (0.331). In PC 3 , highest positive loading was noted for panicle number (0.792) followed by panicle length $(0.432)$ and panicle angle $(0.256)$ with a variance of $14.28 \%$ (Table 4). The initial 2 axis of principal components (PC1 and PC2) captures $52.97 \%$ of total variation. The scatter plot was drawn between two principal components represented a clear pattern of grouping of finger millet cultivars into four quarters (Fig. 2). Based on biplot analysis, four traditional finger millet viz., 'Lala', 'Biri', 'Kurkuti' and 'Chili' were present in one quarter along with hybrid variety 'ML 365' and differentiated from other on the basis of leaf area and panicle weight. In another quarter five local genotypes such as 'Chillikangra', 'Jama', 'Jhana', 'Limca' and 'Telugu' along with hybrid variety 'Bhairabi' and usefully separated from other based upon panicle length and yield parameters. Similarly, based on panicle angle five local finger millet genotypes such as 'Dasara', 'Kalua', 'Muskuri', 'Mami' and 'Ladu' along with 'GPU 66' were separated. In quarter fourth, six traditional finger millet cultivars viz., 'Bada', 'Bhadi', 'Kalia', 'Murda', 'Sillijanha' and 'Taya' were successfully separated from other for the panicle number. 
Table 5

Principal component analysis (PCA) of different morphological traits in studied finger millet genotypes of Koraput.

\begin{tabular}{|llllllll|}
\hline Parameters & PC 1 & PC 2 & PC 3 & PC 4 & PC 5 & PC 6 & PC 7 \\
\hline Panicle number & -0.277 & -0.278 & 0.792 & 0.118 & -0.032 & -0.344 & 0.292 \\
\hline Panicle length & 0.390 & -0.274 & 0.432 & -0.319 & 0.260 & 0.622 & -0.174 \\
\hline Panicle angle & -0.190 & 0.726 & 0.256 & 0.339 & 0.127 & 0.442 & 0.213 \\
\hline Leaf area & 0.442 & 0.331 & 0.173 & 0.090 & 0.531 & -0.519 & -0.325 \\
\hline Leaf weight & 0.588 & 0.005 & -0.112 & -0.010 & -0.025 & -0.060 & 0.798 \\
\hline Panicle weight & 0.424 & 0.159 & 0.245 & 0.215 & -0.772 & -0.018 & -0.303 \\
\hline Yield & 0.114 & -0.431 & -0.132 & 0.845 & 0.191 & 0.165 & -0.071 \\
\hline \% variance & 38.069 & 16.908 & 14.285 & 13.830 & 9.010 & 6.497 & 1.400 \\
\hline Eigen value & 2.665 & 1.184 & 1.000 & 0.968 & 0.631 & 0.455 & 0.098 \\
\hline
\end{tabular}

\section{Relationship Among Studied Panicle Traits}

Relationship among different panicle traits in studied finger millet were carried out by multiple correlation analysis (Fig. 3). Based on the results, panicle length was positively associated with leaf area, leaf weight, panicle weight and yield and negatively related with panicle number and panicle angle (Fig. 3). In addition, on the basis of Pearson's correlation coefficient, panicle number was positively correlated with panicle length $(r=0.997)$, panicle angle $(r=$ $0.725)$, leaf area $(r=0.298)$ and panicle weight $(r=0.532)$ (Table 6). Grain yield in finger millet was positively related with panicle length $(r=0.933)$, panicle angle $(r=0.531)$, leaf area $(r=0.843)$, leaf weight $(r=0.439)$ and panicle weight $(r=0.654)$ (Table 6). 
Table 6

Relationship between different morphological traits in studied finger millet genotypes of Koraput. *: significance at $\mathrm{P}<$ 0.05 , **: significance at $P<0.01$. ns: non-significant.

\begin{tabular}{|c|c|c|c|c|c|c|c|}
\hline Parameters & $\begin{array}{l}\text { Panicle } \\
\text { number }\end{array}$ & $\begin{array}{l}\text { Panicle } \\
\text { length }\end{array}$ & $\begin{array}{l}\text { Panicle } \\
\text { angle }\end{array}$ & $\begin{array}{l}\text { Leaf } \\
\text { area }\end{array}$ & $\begin{array}{l}\text { Leaf } \\
\text { weight }\end{array}$ & $\begin{array}{l}\text { Panicle } \\
\text { weight }\end{array}$ & Yield \\
\hline $\begin{array}{l}\text { Panicle } \\
\text { number }\end{array}$ & 1.000 & $0.997 * \star$ & $0.725^{\star \star}$ & $0.298 *$ & $0.017^{n s}$ & $0.532^{\star}$ & $0.935^{\star \star}$ \\
\hline $\begin{array}{l}\text { Panicle } \\
\text { length }\end{array}$ & & 1.000 & $0.188^{n s}$ & $0.107^{n s}$ & $0.009 \mathrm{~ns}$ & $0.161^{\mathrm{ns}}$ & $0.933^{* *}$ \\
\hline Panicle angle & & & 1.000 & $0.766^{n s}$ & $0.132^{n s}$ & $0.938 * \star$ & $0.531 *$ \\
\hline Leaf area & & & & 1.000 & $0.001^{n s}$ & $0.074^{n s}$ & $0.843^{\star *}$ \\
\hline Leaf weight & & & & & 1.000 & $0.001^{n s}$ & $0.439 *$ \\
\hline $\begin{array}{l}\text { Panicle } \\
\text { weight }\end{array}$ & & & & & & 1.000 & $0.654^{\star \star}$ \\
\hline Yield & & & & & & & 1.000 \\
\hline
\end{tabular}

\section{Cluster analysis based on panicle traits in finger millet genotypes}

The genotypic relationship among 20 indigenous finger millet genotypes and three hybrid cultivars was evaluated through Bray-Curtis similarity index (Fig. 4). Based on studied morphological traits, finger millet genotypes clustered into 2 large clusters and different sub-clusters. The largest cluster consists of 15 finger millet genotypes with more than $88 \%$ similarity which consisting of 13 indigenous genotypes (Dasara, Kalua, Ladu, Muskuri, Biri, Bada, Chilli, Kurkuti, Lala, Limca, Murda, Mami and Bhadi) and 2 hybrid variety (GPU 66 and ML 365). The hybrid variety 'GPU 66' showed more than 95\% similarity with 'Kalua' and 'Dasara' and present in a small sub group. Similarly, in another major cluster, seven indigenous finger millet genotypes along with hybrid variety 'Bhairabi' showed more than $90 \%$ similarity (Fig. 4).

\section{Discussion}

Genetic variability study of germplasm provides fundamental information concerning genetic characteristics of the population based upon which breeding techniques are developed (Umar and Kwon-Ndung 2014). This is the first report on genotypic variability of panicle traits in traditional finger millet genotypes from Koraput valley of Eastern Ghats. The present investigation analyzed panicle phenotyping in 20 traditional finger millet genotypes and compared with 3 hybrid cultivars (GPU66, ML365 and Bhairabi) popularly cultivated in the locality. The analysis of variance clearly indicated that high significant difference of panicle traits viz., panicle length, panicle number, panicle weight, panicle angle and grain yield were recorded across the examined finger millet genotypes. Current findings also exhibited higher $\mathrm{CV} \%$ of different panicle traits were observed among the studied finger millet genotypes. Thus, it is indicated that there was sufficient variation of panicle characteristics in the studied genotypes, which provide ample scope for selecting superior genotypes and could be utilized in future breeding programs. Similarly, physiological variability in different underutilized finger millets from various parts of India also reported earlier by Panda et al. (2020b), Pradhan et al. (2019) and, Kumari and Singh (2015). 
The panicle architecture is a key component which enhances grain productivity and basically controlled by panicle number, panicle length, accompanying branching pattern and grains (Huang et al. 2018; Patil et al. 2018). Certain traditional finger millet genotypes like Bhadi, Mudra, Telgu and Chilli exhibited significantly higher panicle number and panicle weight in compassion to rest genotypes that determined superiority of panicle traits. Panicle length was highest in 'Chillikangra' and highest panicle angle was found in 'Dasara' and 'Kalua' genotypes in comparison to rest genotypes. Wang et al. (2008) reported the shape, size and angle of panicle is crucial for arrangement of grains in the panicle and also helpful for grain filling in cereal crops. There were significant variability of leaf area and leaf weight observed among finger millet genotypes and maximum leaf area and leaf weight was observed in hybrid variety $\mathrm{ML}$ 365. It is generally advocated that larger leaf area increases the photosynthesis capacity and improve the dry matter and ultimately helpful for better panicle growth (Yoshinaga et al. 2013). Yield is most significant complex traits in finger millet that is affected by numerous genetic and environmental elements (Ulaganathan and Nirmalakumari 2013). In the current experiment significant variability of grain yield was recorded among the finger millet genotypes. Though the hybrid varieties Bhairabi and ML 365 showed highest yield but some of the traditional genotypes such as 'Limca' and 'Kalia' showed better yield at par with the hybrids. Similar results of morphological variations in finger millets are also reported in indifferent germplasm collections (Shet et al. 2009; Ulaganathan and Nirmalakumari 2013; Patil et al. 2018). To know the relationship of grain yield with other panicle traits, data are subjected to multiple correlation analysis. Results indicated that grain yield in studied finger millet genotypes were positively associated with panicle number, panicle length, leaf weight, leaf area, and panicle angle and panicle weight. These findings are reliable with the previous literature of Reddy et al. (2013); Ulaganathan and Nirmalakumari (2013) and Wolie et al. (2013) that grain weight and harvest index depend on the shape, size and number of panicles. Kumari and Singh (2016) reported that yield of finger millet relies upon different growth and yield characteristics like filled grain number, panicle number and grain weight. In contrast, it was also reported that variation of grain yield was not necessarily associated with leaf area in cereals like finger millet (Panda et al. 2020b) and rice (Subrahmanium 2000; Panda et al. 2020c).

In the present study, panicle angle, leaf area and panicle weight showed the higher PCV and GCV value indicating these traits are more variable in the germplasm. The PCV value was greater than GCV and ECV for panicle traits and also interesting to note that the variation among GCV and PCV were minimum in all traits. This implies about following traits were least affected by environment and additive gene effects indicating genotypes can be improved based on genotypic values (Reddy et al. 2013). Similar results were also published by Wolie et al. (2013) and Kumari and Singh (2015) in different finger millet accessions. High level of heritability and genetic advance will be helpful for selection in crop improvement (Sahu et al. 2017; Panda et al. 2020c). The genotypes under study showed high broadsense of heritability value for grain yield (82.27\%) and panicle weight (75.96\%) compared to other traits. It indicates that following traits are less impacted by environment and effective for selection on the basis of phenotypic performance. The following results are consistent with previous report of Reddy et al. (2013) and, Kumari and Singh (2015) that higher value of heritability can be used for selection criteria for finger millet improvement program. In the present study GAM value changed from 11.01-29.26\% and high GAM was recorded for panicle angle, leaf area, panicle weight, panicle number and yield. It is suggested that these traits can be used as selection criteria in improving grain yield in crop improvement program.

Principal component analysis reveals the pattern of genotypic variation and useful for identifying the major contributing traits for phenotypic variability (Mishra et al. 2019). In the light of the outcomes, first two principal components capture $52.97 \%$ of total variation and highest positive loading was observed for leaf weight followed by panicle number, panicle weight and panicle length and leaf area. It suggested that these parameters are the major determinant for phenotypic diversity in studied genotypes compared to other traits. Similar results of phenotypic diversity of morpho-physiological traits are also proclaimed in traditional rice landraces (Panda et al. 2020c) and in 
underutilized millets (Panda et al. 2020b) from Koraput. Based on panicle attributes, genotypic relationship among the studied finger millet genotypes were evaluated by cluster analysis (Fig. 4). Bray-Curtis similarity index classified the genotypes in two major clusters and different sub-clusters. The largest cluster consists of 15 genotypes with more than $88 \%$ similarity which consisting of 13 indigenous genotypes and two hybrid variety. Higher similarity among the genotypes may be because of their similar origin, ecotype and cultivated only in a particular region. Results also recorded that no duplicate genotypes existed and indicated the considerable genetic difference between the genotypes for panicle traits. Three indigenous finger millet genotypes such as Taya, Kalua and Dasara were having maximal similarity index along with hybrid cultivars GPU 66 and Bhairabi. The genetic variability results in indigenous finger millet genotypes may provide baseline information for conservation and could be used for varietal improvement of future breeding programmes.

\section{Conclusion}

The present study revealed existence of huge variability of panicle traits in studied finger millet genotypes, which provide ample scope for selecting superior genotypes for future breeding programs. Principal component analysis revealed that panicle number, panicle length, panicle weight and leaf area are the major contributing traits for phenotypic variability between the examined genotypes. Some traditional finger millet genotypes like Bhadi, Murda, Telgu and Chilli revealed superiority of panicle traits and Limca and Kalia showed better grain yield at par with the hybrids, which can be utilized in the future crop improvement program. Higher level of heritability and genetic advance were observed for panicle angle, leaf area, panicle weight, panicle number and yield, which suggested that these characters may be helpful for selection in finger millet improvement programs. Further research on population structure, QTL mapping and allele diversity in these genotypes are required for use in future breeding program.

\section{Declarations}

Conflict of interest: The authors declare that they have no conflict of interest

Funding: This work was supported by Central University of Odisha, Koraput, India

\section{Ethics approval: NA}

Consent to participate: NA

Consent for publication: NA

Availability of data and material: NA

Code availability: NA

Author contributions: DP and AT designed the experiments; KL cultivated the plants and maintained the experimental field. AT, AP and PKB performed the measurement of panicle traits. DP analyzed the data and wrote the paper. All authors read and provided helpful discussions for the manuscript.

\section{References}

Babu BK, Dinesh P, Agrawal PK, Sood S, Chandrasekhar C (2014) Comparative genomics and association mapping approaches for blast resistant genes in finger millets using SSRs. PLoS One 9(6):e99182 
Babu GA, Vinoth A, Ravindhran R (2018) Direct shoot regeneration and genetic fidelity analysis in finger millet using ISSR markers. Plant Cell Tissue Organ Cult 132:157-164

Burton GW, Devane EH (1953) Estimating heritability in tall fescue (Festuca arundinacea) from replicated clonal material. Agron J 45(10):478-481

Ceasar SA, Maharajan T, Krishna TPA, Ramakrishnan M, Roch GV, Satish L, Ignacimuthu S (2018) Finger millet [Eleusine coracana (L.) Gaertn.] improvement:Current status and future interventions of whole genome sequence. Front Plant Sci 9:1054

Chandra D, Chandra S, Pallavi A, Sharma K (2016) Review of finger millet (Eleusine coracana (L.) Gaertn): A power house of health-benefiting nutrients. Food Sci Human Well 5(3):149-155

Dida MM, Wanyera N, Harrison DMLN, Bennetzen JL, Devos KM (2008) Population structure and diversity in finger millet (Eleusine coracana) germplasm. Trop Plant Biol 1:131-141

Falconer DS, Mackay TFC (1996) Introduction to quantitative genetics, 4th edn. Longmans Green, Harlow

Gimode D, Odeny DA, De Villierls EP, Wanyonyi S, Dida MM, Mneney EE, Muchugi A, Machuka J, De Villiers SM (2016) Identification of SNP and SSR markers in finger millet using next generation sequence technology. PLoS One 11(7):e0159437

Gupta V, Jata PK, Verma R, Kothari SL, Kachhwaha S (2017) Nickel accumulation and it's effect on growth, physiological and biochemical parameters in millets and oats. Environ Sci Poll Res 24(30):23915-23925

Huang Y, Bai X, Luo M, Xing Y (2018) Short Panicle 3 controls panicle architecture by upregulating APO2/RFL and increasing cytokinin content in rice. J Integr Plant Biol 61(9):987-999

Johnson HW, Robinson HF, Comstock RE (1955) Estimates of genetic and environmental variability in soybeans. Agron J 47(7):314-318

Kumar A, Yadav S, Panwar P, Gaur VS, Sood S (2015) Identification of anchored simple sequence repeat markers associated with calcium content in finger millet (Eleusine coracana). Proc Natl Acad Sci India Sect B Biol Sci 85:311317

Kumari S, Singh KS (2015) Assessment of genetic diversity in promising finger millet [Eleusine coracana (L.) Gaertn] genotypes. Suppl Genet Plant Breed 10(2):825-830

Kumari S, Singh SK (2016) Correlation and path coefficient analysis for yield and its yield attributes in promising finger millet (Eleusine coracana (L.) Gaertn) genotypes. Suppl Genet Plant Breed 11(2):1079-1082

Lei Q, Zhou J, Zhang W, Luo J, Wu K, Long C (2018) Morphological diversity of panicle traits in Kam fragrant glutinous rice (Oryza sativa). Genet Resour Crop Evol 65:775-786

Mishra SS, Behera PK, Panda D (2019) Genotypic variability for drought tolerance-related morpho physiological traits among indigenous rice landraces of Jeypore tract of Odisha, India. J Crop Improv 33:254-278

Mutch DM, Wahli W, Williamson G (2005) Nutrigenomics and nutrigenetics: the emerging faces of nutrition. FASEB J 19:1602-1616 
Panda D, Sahu N, Behera PK, Lenka K (2020c) Genetic variability of panicle architecture in indigenous rice landraces of Koraput region of Eastern Ghats of India for crop improvement. Physiol Mol Biol Plants 26(10):1961-1971

Panda D, Sailaja NH, Behera PK, Lenka K, Sharma SS, Lenka SK (2020b) Genetic diversity of underutilized indigenous finger millet genotypes from Koraput, India for crop improvement. J Plant Biochem

Biotechnol. https://doi.org/10.1007/s13562-020-00557-w

Panda D, Sailaja NH, Padhan B, Lenka, K (2020a) Sprouting-associated changes in nutritional and physic functional properties of indigenous millet from Koraput, India, Proc Natl Acad Sci India Sect B Biol Sci 90(1):79-86

Patil SK, Gupta SK, Dang KS, Sashibhusan D, Balaram M, Ramesh T (2018) Panicle traits and plant heights are important selection indices to enhance productivity in pearl millet (Pennisetum glaucum) populations. Int $\mathrm{J}$ Curr Microbiol Appl Sci 7(12):306-312

Pradhan A, Panda AK, Bhavani RV (2019) Finger millet in tribal farming systems contribute to increased availability of nutritious food at household level: insight from India. Agric Res 8:540-547

Rajendran HAD, Muthusamy R, Stanislaus AC, Krishnaraj T, Kuppusamy S, Ignacimuthu S, Al-Dhabi NA (2016) Analysis of molecular variance and structure in Southern Indian finger millet genotypes using three different molecular markers. J Crop Sci Biotechnol 19(4):275-283

Ramakrishnan M, Ceasar SA, Duraipandiyan V, Al-Dhabi NA, Ignacimuthu S (2016) Using molecular markers to assess the genetic diversity and population structure of finger millet (Eleusine coracana (L.) Gaertn.) from various geographical regions. Genet Resour Crop Evol 63:361-376

Reddy CV, Reddy PVRM, Munirathnam P, Gowda J (2013) Studies of genetic variability in yield and yield attributing traits of finger millet [Eleusine coracana (L.) Gaertn]. Indian J Agric Res 47(6):549-552

Saha D, Gowda MVC, Arya L, Verma M, Bansal KC (2016) Genetic and genomic resources of small millets. Crit Rev Plant Sci 35:56-79

Sahu P, Sharma D, Mondal S, Kumar V, Singh S, Baghel S, Tiwari A, Vishwakarma G, Das BK (2017) Genetic variability for grain quality traits in indigenous rice landraces of Chhattisgarh, India. J Exp Biol Agricul Sci 5(4):439-455

Shet RM, Gireesh C, Jagadeesha N, Lokesh GY, Gowda J (2009) Genetic variability in segregating generation of interspecific hybrids of finger millet (Eleusine coracana (L.) Gaertn.). Environ Ecol 27(3):1013-1016

Sood P, Singh RK, Prasad M (2019) Millets genetic engineering: the progress made and prospects for the future. Plant Cell Tissue Organ Cult 137:421-439

Steel RG, Torrie JH, Dickey DA (1997) Principles and procedures of statistics: A biological approach.McGraw-Hill, New York

Subrahmanyam D (2000) Genotype variability in photosynthetic characteristic in finger millet. Photosynthetica 38(1):105-109

Ulaganathan V, Nirmalakumari A (2013) Phenotypic selection of elite genotypes based on genetic analysis in finger millet [Eleusine coracana (L.) Gaertn] germplasm. Plant Arch 13(2):1003-1008 
Umar ID, Kwon-Ndung EH (2014) Assessment of variability of finger millet (Eleusine coracana (L.) Gaertn) landraces germplasm in Northern Nigeria. Niger J Genet 28:48-51

Wakista PW, Dasanayaka PN, Illeperuma RJ, Perera SACN (2017) Assessment of genetic diversity of a representative sample of finger millet (Eleusine coracana (L.) Gaertn.) collected from four different district of Sri Lanka using SSR markers. Sri Lankan J Biol 2(1):1-8

Wang F, Chang F, Zhang G (2008) Impact of cultivar variation in density of rice panicle on grain weight and quality. J Sci Food Agric 88:897-903

Wolie A, Belete K, Dessalegn T (2013) Heritability, variance components and genetic advance of some yield and yield related traits in Ethiopian collections of finger millet (Eleusine coracana (L.) Gaertn.) genotypes. Afr J Biotechnol 12(36):5529-5534

Wu Y, Huang M, Tao X, Guo T, Chen Z, Xiao W (2016) Quantitative trait loci identification and meta analysis for rice panicle-related traits. Mol Genet Genom 291(5):1-14

Yoshida S, Forno DA, Cock JH, Gomez KA (1976) Laboratory Manual for Physiological Studies of Rice. IRRI,Phillippines

Yoshinaga S, Takai T, Sanoh YA, Ishimaru T, Kondo M (2013) Varietal differences in sink production and grain filling ability in recently developed high-yielding rice (Oryza sativa L.) varieties in Japan. Field Crops Res150:74-82

Zhao M, Sun J, Xiao Z, Cheng F, Xu H, Tang L, Chen W, Xu Z, Xu Q (2016) Variations in Dense and Erect Pnicle 1 (DEP1) contribute to the diversity of the panicle trait in high yielding japonica rice varieties in northern China. Breed Sci 66:599-605

Zhou X, Xia Y, Ren X, Chen Y, Huang L, Huang S, Liao B, Lei Y, Yan L, Jiang H (2014) Construction of a SNP based genetic linkage map in cultivated peanut based on large scale market development using next generation double-digitrestriction-site-associated DNA sequencing (ddRADseq). BMC Genomics 15(1): 351

\section{Figures}




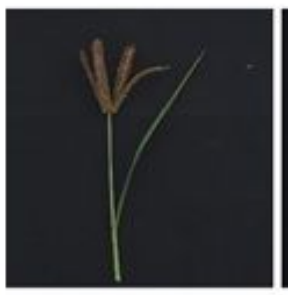

BADA

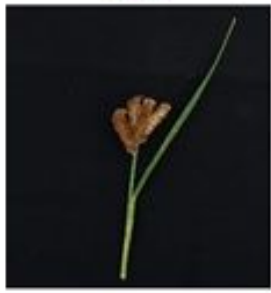

DASARA

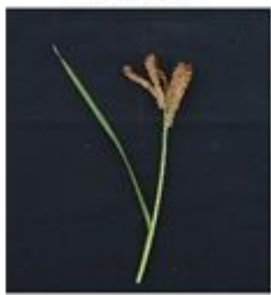

KURKUTI

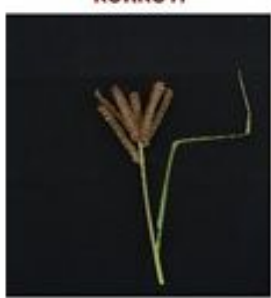

MURDA

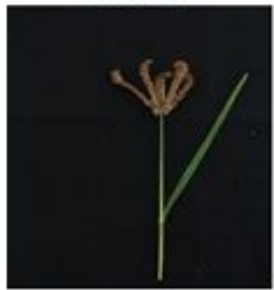

GPU 66 (Hvbrid)

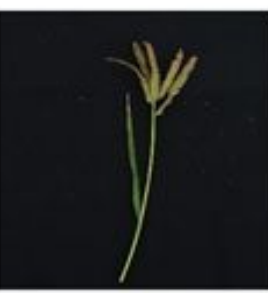

BHADI

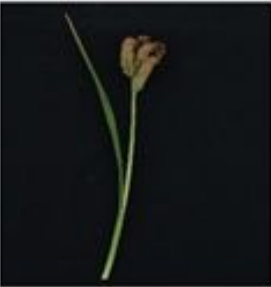

JAMA

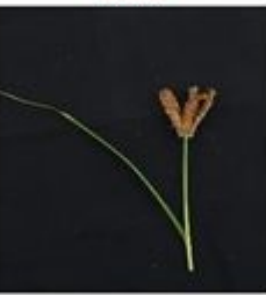

LADU

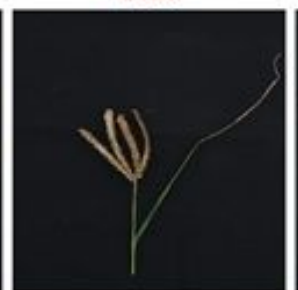

MUSKURI

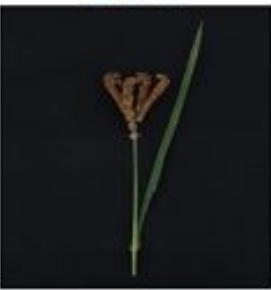

ML 365 (Hvbrid)

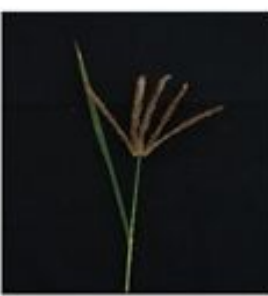

BIRI

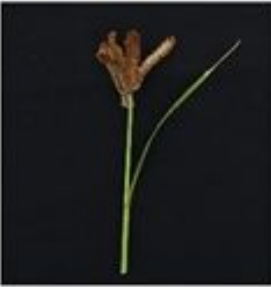

JHANA

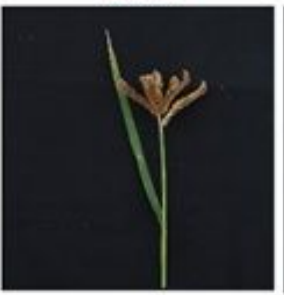

LALA

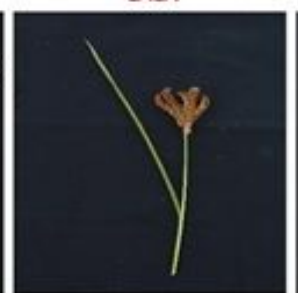

SILLIJANHA

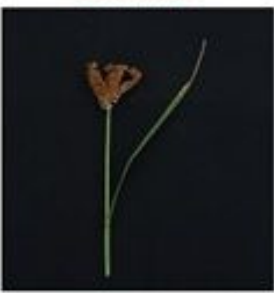

BHAIRABI (Hvbrid)

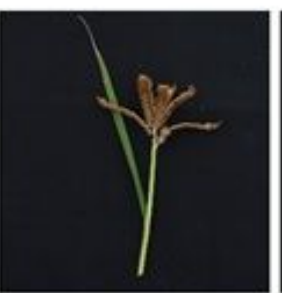

CHILII

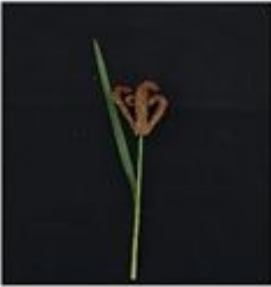

KALIA

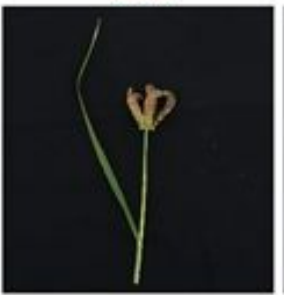

UIMCA

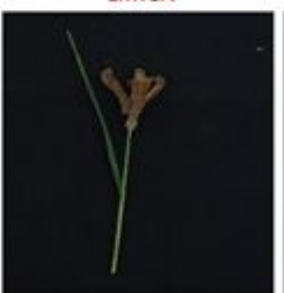

TAYA

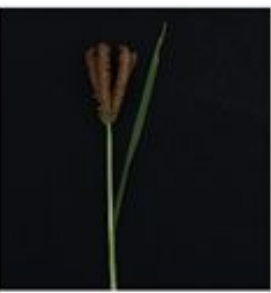

CHILLI KANGRA

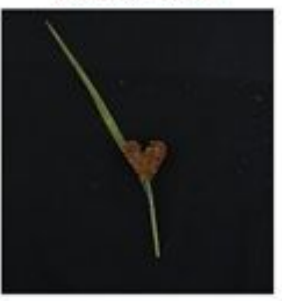

KALUA

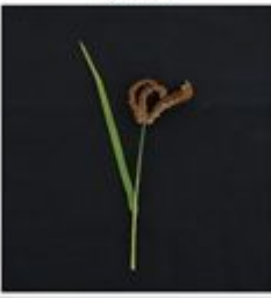

MAMI

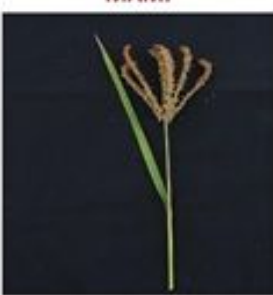

TELUGU

Figure 1

Morphological diversity of panicles in studied finger millet genotypes of Koraput. 


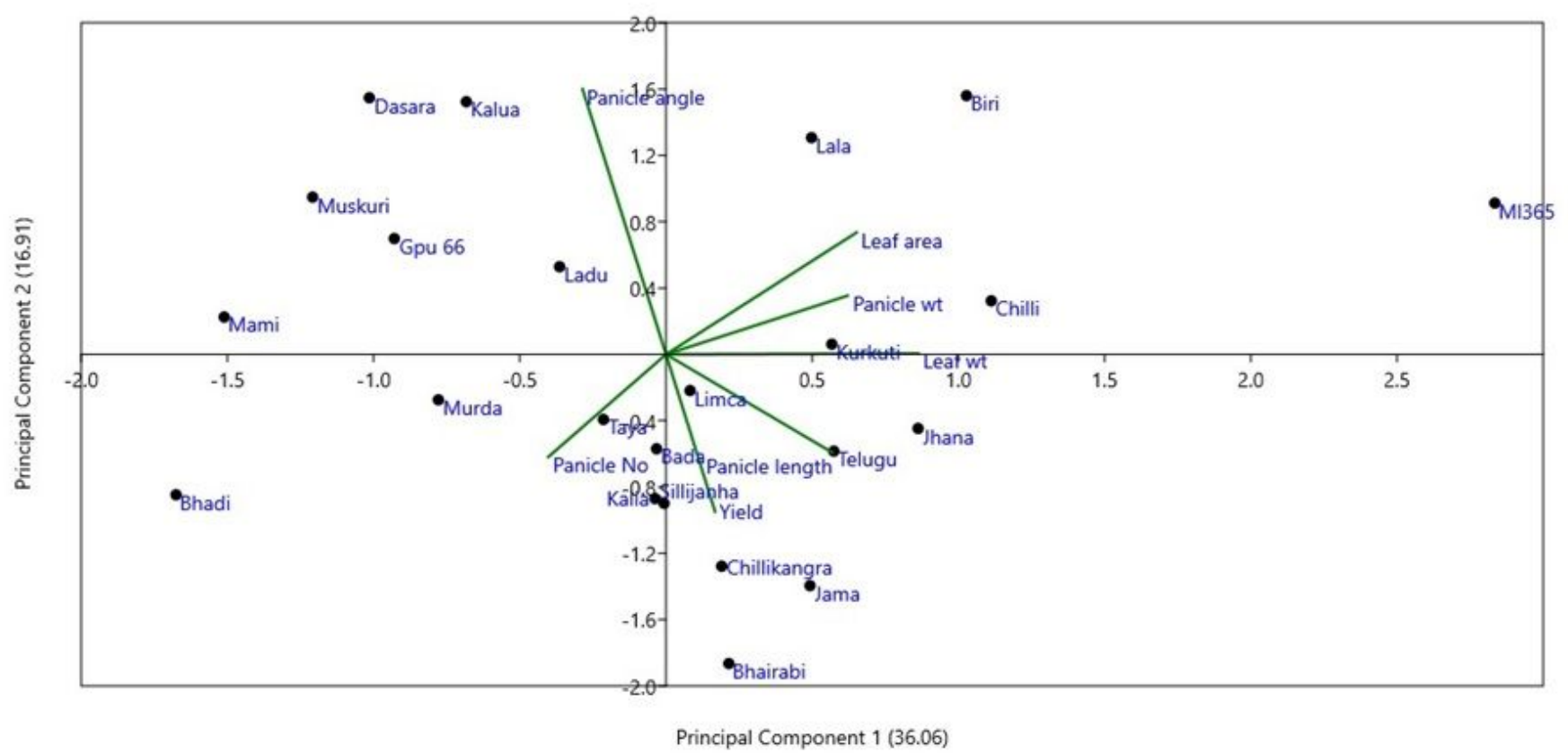

\section{Figure 2}

Scatter plot showing the genotypic relationship in studied finger millet genotypes of Koraput on the basis of different panicle traits.

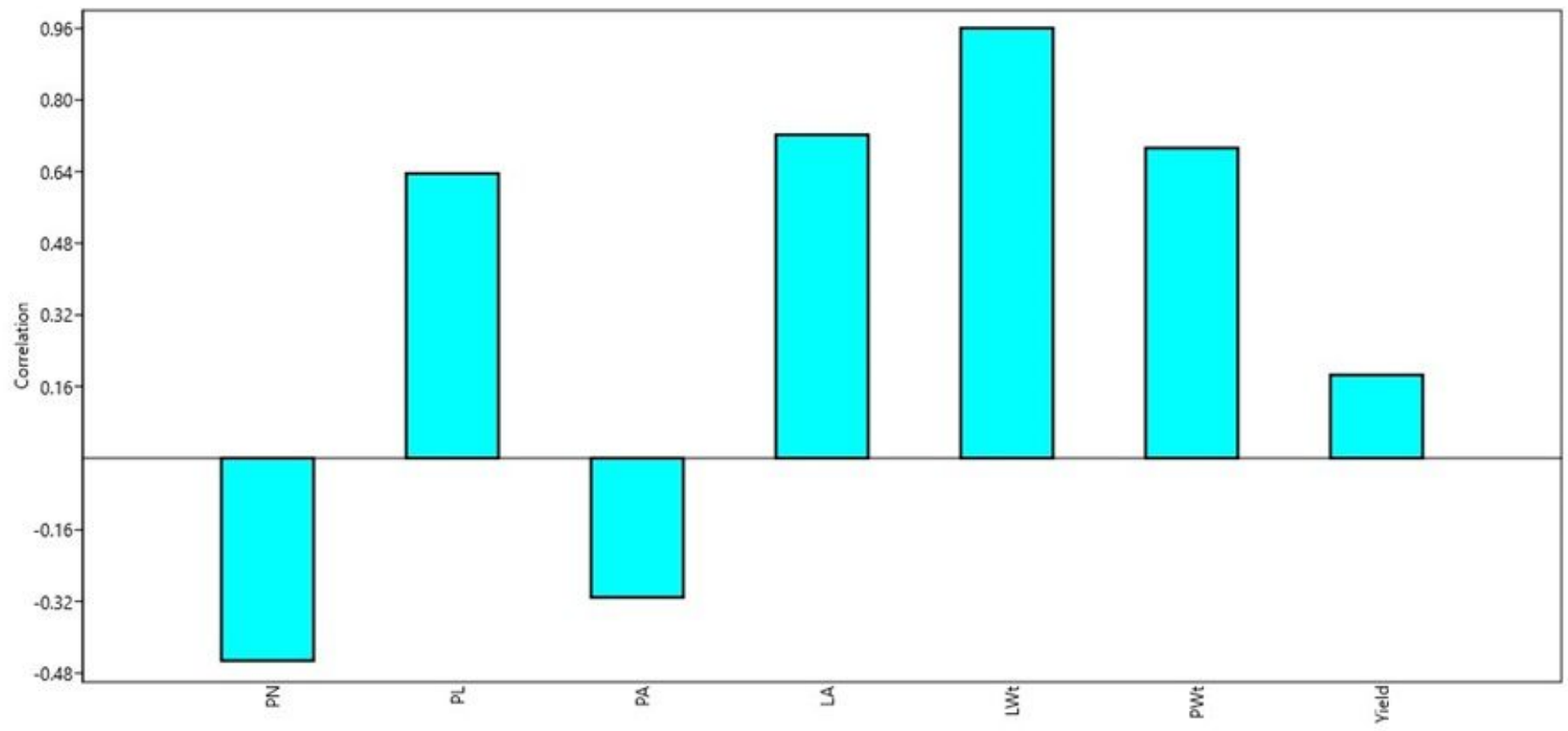

Figure 3

Relationship of different panicle traits in studied finger millet genotypes of Koraput. 


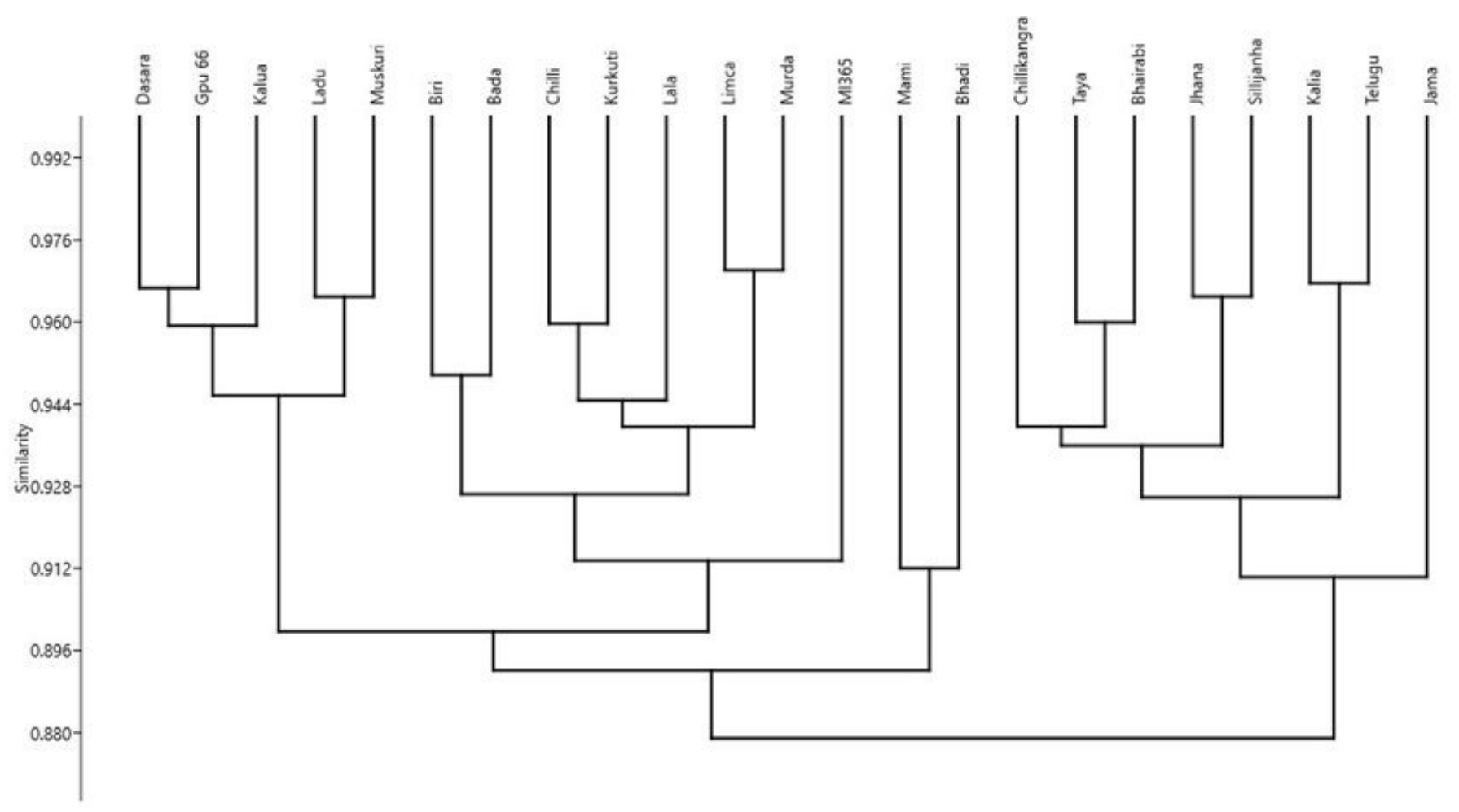

Figure 4

Dendrogram showing the genotypic relationship for different finger millet genotypes of Koraput based on panicle traits. 